\title{
Measurement of the Damping of Liquid Surface Wave by Diffraction Method
}

\author{
Jun Dong \\ Department of Electronics and Information Engineering, \\ Xi'an Institute of Posts and Telecommunications, Xi'an 710121, People's Republic of China \\ Jianxia Qi \\ Department of Applied Mathematics \& Applied Physics, \\ Xi'an Institute of Posts and Telecommunications, Xi'an 710121, People's Republic of China \\ Runcai Miao \\ Institute of Physics and Information Technology, Shaanxi Normal University, Xi'an 710062, People's Republic of China
}

Received on 12 April, 2007

\begin{abstract}
A simple method for measuring the damping of the liquid surface wave (LSW) based on the diffraction method was proposed in this paper. In the experiment, the phenomenon was observed that the intensity distribution of the diffraction patterns from the LSW varies with the position of the incident spot. By theoretical analysis of the relationship between the intensity distribution and the LSW amplitude, the damping constant was obtained. In addition, the viscosity of the liquid can be calculated with this method, too.
\end{abstract}

Keywords: Diffraction; Liquid surface wave; Damping constant

\section{INTRODUCTION}

In recent years, the study of liquid surfaces has attracted great interest both in labs and in industries [1-4]. Many techniques have been applied to investigate the characteristics of the liquid surface wave (LSW), which provide us a large amount of information about the liquid. Among these techniques, most are used to study the surface tension, the relation of dispersion, the phase velocity of LSW and so on [58]. Besides, there are also a few researches focusing on the liquid surface wave damping $[9,10]$. For instance the laserscanning slope technique is applied to study the LSW at a low frequency (a few hertz), because the LSW wavelength is much greater than the scanning laser diameter in the case [1114]. The imaging analyzer is used to study LSW at a few tens hertz, and the image is formed by the light transmitted through the dyed liquid medium $[15,16]$. The Capacitive Antenna method $[17,18]$ is used to measure surface wave amplitude simultaneously at two radii from the wave drive point, but the needed equipments are complicated. Besides, the surface light scattering method is very effective to study the LSW [1924]. However, the usefulness of light scattering is limited because it is most suitable for studying LSW with wavelength in the sub micrometer range. In addition, the corresponding data from light scattering is greatly affected by the reduction of surface tension at the short wavelengths associated with thermally excited waves. Apart from the limitation of the technique, the equipment for the surface light scattering method is also complex and expensive [25-28].

However, there are few experiments performed to study the transparent liquid surface wave damping at a few hundreds hertz [29,30]. In our former investigation [31], the diffraction method was used to study the low-frequency liquid surface waves, and the disappearance of the zero-order diffraction fringe was experimentally observed, which corresponds to $100 \%$ diffraction efficiency.
In this letter, not only the stationary diffraction patterns were obtained, but also the phenomenon that the intensity distribution of the diffraction patterns varies with the shift of the incident spot was observed experimentally. By theoretical analyzing the influence of the amplitude of LSW on the intensity distribution of the diffraction patterns, the relationship between the amplitude of LSW and the distance between the exciter and the spot could be obtained. So based on the analysis of the intensity distribution resulting from LSW at different positions, a simple technique for measuring the damping constant of liquid surface wave at several hundreds hertz was proposed. Compared with the techniques aforementioned, the diffraction method presented in this letter can be applied whether the liquid is transparent or not, because the transmission light can only carry the information of LSW in the dyed liquid, whereas the diffraction light does not has this limitation. In addition, the latter technique can be easily implemented and the experimental results can be well repeated.

\section{EXPERIMENT SETUP AND DESCRIPTION}

The depiction of the experimental setup is shown schematically in Fig. 1. A low frequency signal generator produces an output at a few hundreds Hertz, and then drives the LSW exciter. The wave exciter is a triangle delicate metal frame and its plane is perpendicular to the liquid surface. A laser beam is divided into two sub-beams by a beam splitter. One is used to monitor the laser output stability, and the other is directly incident upon the liquid surface where the LSW is traveling. For reference, the diameter of the laser beam cross-section is about $1.1 \mathrm{~mm}$. For the oblique incidence of the laser beam, the shape of the illuminating area on the liquid surface is an ellipse whose major axis and minor axis are about 5.8 and $1.1 \mathrm{~mm}$, respectively. The major axis is parallel to the LSW traveling direction. In our experiment, the incident angle is 
$1.38 \mathrm{rad}$ and the laser wavelength is $473.0 \mathrm{~nm}$, respectively. The liquid sample cell is put on a special plate, whose position can be adjusted. The exciter is installed on a movable holder so that the relative distance between the acoustic source and the light incident point can be changed. A CCD imager is used to detect the reflected light from the liquid surface with a traveling acoustic wave. The distance between the light incident point and the observation plane is about $8.56 \mathrm{~m}$. Because this distance is far enough, the Fourier-transform lens is not used in our experiment setup. The diffraction pattern is subsequently displayed, stored, and processed by a computer. The experiment was conducted at the room temperature $25^{\circ} \mathrm{C}$ and the liquid sample was distilled water.

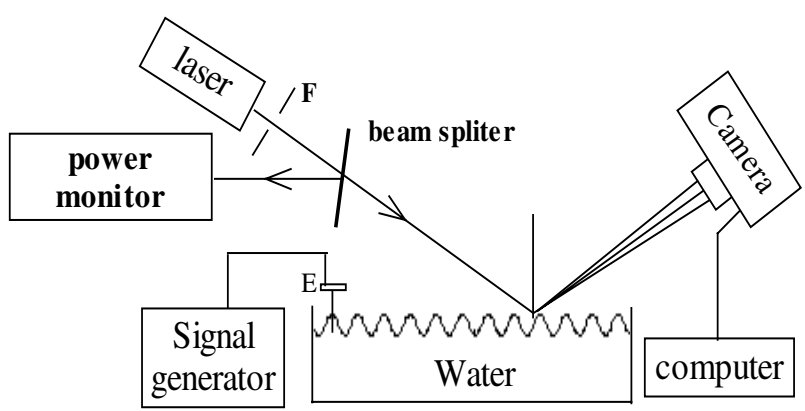

FIG. 1: Schematic diagram of experimental setup E: exciter, M: mirror, P: polarizer, F: filter.

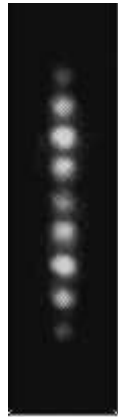

(a)

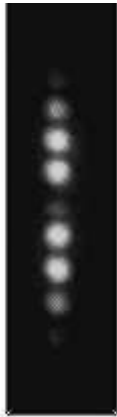

(b)

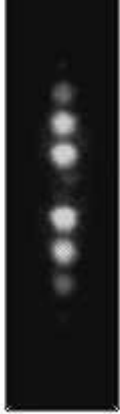

(c)

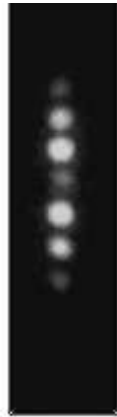

(d)

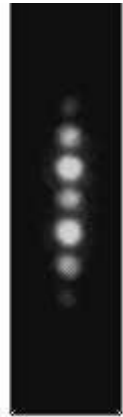

(e)

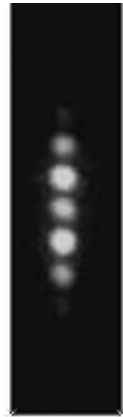

(f)
FIG. 2: Diffraction patterns from the LSW at $230 \mathrm{HZ}$ on a water-air surface with different distance between the exciter and the light spot (a) $5.0 \mathrm{~cm}$, (b) $5.5 \mathrm{~cm}$, (c) $6.0 \mathrm{~cm}$, (d) $6.5 \mathrm{~cm}$, (e) $7.0 \mathrm{~cm}$, (f) $7.5 \mathrm{~cm}$.

In our experiment, at some fixed frequency, we change the distance between the incident spot and the exciter by moving the exciter, and then obtain the diffraction patterns. And Fig. 2 is obtained at a frequency of $230 \mathrm{~Hz}$. When the distance is about $5.0 \mathrm{~cm}$, we get the diffraction pattern shown in Fig. 2(a). Then, with the shift of the exciter, we get the diffraction patterns at different positions, which are shown in Fig. 2 (b), (c), (d), (e), (f), and the distance is about $5.5 \mathrm{~cm}$,
$6.0 \mathrm{~cm}, 6.5 \mathrm{~cm}, 7.0 \mathrm{~cm}$, and $7.5 \mathrm{~cm}$. By observing the intensity distribution of the diffraction pattern, one may easily find that the intensity distribution varies with the position of the illuminated spot. Due to the viscosity [32], the amplitude of LSW decreases along the wave propagation. That is, the intensity distribution of diffraction patterns on the reflected direction varies with the amplitude of LSW. So it is possible to study the relationship of the LSW amplitude and the traveling distance by analyzing the intensity distribution of the diffraction patterns.

\section{THEORETICAL ANALYSIS}

Though the surface particle motion is actually somewhat more complex in nature, LSW propagation can be adequately approximated as a traveling sinusoidal disturbance [33], as shown in Fig. 3.

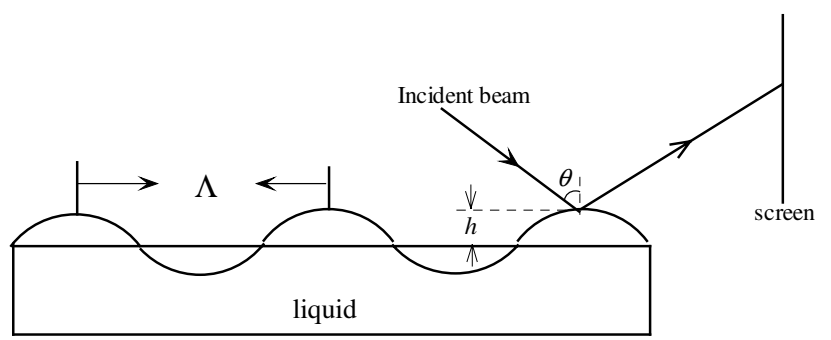

FIG. 3: The LSW geometry.

With careful inspection of Fig. 3, one may readily determine that the phase modulation impressed upon a laser beam upon reflection from the liquid surface can be written as [34]

$$
\phi(x)=\frac{2 \pi}{\lambda}[(2 h \cos \theta) \sin (\Omega t-k x)]
$$

where $h$ is the LSW amplitude, $\theta$ is the angle of incidence, $\lambda$ is the free-space wavelength of the illumination beam, $\Omega$ is the LSW frequency, $x$ is a one-dimensioned position variable along the surface, $k$ is the one-dimensioned acoustic-wave vector, and $k=\frac{2 \pi}{\Lambda}$, where $\Lambda$ is the LSW wavelength. Note that the factor of 2 in the square bracketed term of Eq. 1 arises because the phase modulation is doubled upon reflection. In our experiment, $\theta=1.38 \mathrm{rad}, \lambda=473.0 \mathrm{~nm}$

In the experiment, the illuminated region is so small that the amplitude $h$ can be approximately assumed as a constant over the region of interest. The light field strength in the region of Fraunhofer diffraction is simply the Fourier transform of the object function, that is, in the case of oblique incidence, given by

$$
\exp \left\{j \frac{2 \pi}{\lambda}[2 h \cos \theta \sin (\Omega t-\vec{K} \cdot \vec{r} / \cos \theta)]\right\}
$$

The corresponding intensity pattern becomes [35] 


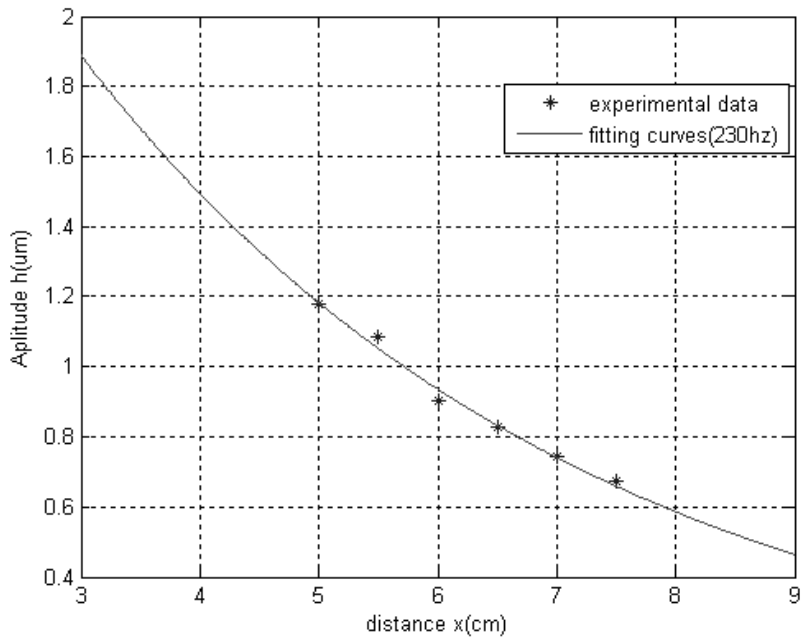

FIG. 4: The LSW amplitude $h$ VS the distance $x$ at $230 \mathrm{~Hz}$.

$$
I\left(x^{\prime}\right)=\sum_{n} J_{n}^{2}(4 \pi h \cos \theta / \lambda) \delta\left(\frac{x^{\prime}}{\lambda z}-\frac{n}{\Lambda \cos \theta}\right),
$$

where $J_{n}$ is Bessel's function of the first kind, order $n, \delta$ represents the delta function, $x^{\prime}$ is the position variable on the observation plane, and $z$ is the observation distance from the liquid surface to the observation plane.

It can be seen from Eq.(3) that the introduction of LSW has deflected energy out of the zero-order component into a multitude of higher orders. The displacement of the $n t h$ order from the center of the diffraction pattern is $\frac{n \lambda z}{\Lambda \cos \theta}$, while the peak intensity of the $n t h$ order is simply determined by $J_{n}^{2}\left(\frac{4 \pi h \cos \theta}{\lambda}\right)$, which is a function of LSW amplitude $h$. It is known from Eq.(3) that the peak intensity will change with the LSW amplitude $h$ for the same order diffraction pattern. Thus, we can determine the LSW amplitude by studying the intensity of the diffraction patterns. With the comparison of the ratio of the intensity for any two or three orders in theory and in experiment, we can determine the LSW amplitude at a fixed position. Then the distance is changed by moving the exciter, and the amplitudes of LSW at different position are obtained with the same method. Therefore, based on the analysis of the intensity distribution of diffraction patterns, the damping of LSW can be studied.

\section{RESULTS AND DISCUSSION}

With the diffraction method, the amplitudes of LSW at $230 \mathrm{~Hz}$ in Fig. 2 are calculated. Fig. 4 shows the relationship of the amplitude $h$ and the distance $x$. In Fig. 4, the discrete dots are the experimental data. The solid curve represents the best fit obtained using the least fits method to the experimental data. One may get the conclusion from Fig. 4 as follows, the amplitude $h$ changes with the distance $x$, and when $x$ is

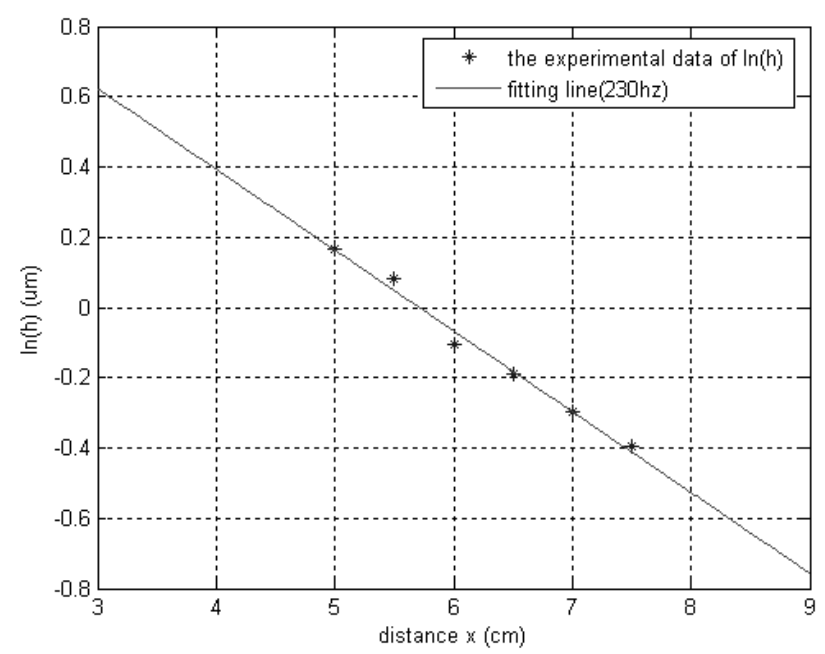

FIG. 5: The logarithm of the amplitude VS the distance at $230 \mathrm{HZ}$.

smaller, i.e., the light spot is closer to the exciter, the amplitude is larger. With the increase of the distance, the amplitude of LSW decreases in the approximately exponential manner along the direction of the wave propagation. The relationship between the amplitude $h$ and the traveling distance $x$ can be written as

$$
h=h_{0} \exp (-\alpha x)
$$

Where $h_{0}$ is the amplitude of the acoustic source, and $\alpha$ is the damping constant. For the simplicity of calculation, the Eq.(4) can be rewritten as

$$
\ln h=\ln h_{0}-\alpha x
$$

the relationship between the $\ln h$ and the distance $x$ is shown in Fig. 5. The fitted line is a straight line. The slope yields the spatial damping coefficient $\alpha=23.0 \mathrm{~m}^{-1}$.

It should be noted that the surface wave attenuation depends upon the wave frequency. The spatial damping constant of the surface wave $\alpha$ is approximately given by the hydrodynamics theory as [36]

$$
\alpha=\frac{8}{3} \frac{\pi \nu \rho f}{\sigma}
$$

where $\sigma$ is the surface tension, $\rho$ is the density of the sample liquid, and $v$ the kinetic viscosity, $f$ is the LSW frequency. Here, the amplitude is assumed to be much smaller than the wavelength. For pure water at $25^{\circ} \mathrm{C}, \mathrm{v}=0.897 \times 10^{-6} \mathrm{~m}^{2} / \mathrm{s}$, $\rho=997.1 \mathrm{~kg} / \mathrm{m}^{3}, \sigma=7.26 \times 10^{-2} \mathrm{~N} / \mathrm{m}$, and $f=230 \mathrm{~Hz}$, it is calculated that $\alpha=23.7 \mathrm{~m}^{-1}$. Comparing the experimental results with the theoretical data, they are in good agreement.

In our experiment, we applied the diffraction method to the LSW damping problem at a few hundreds hertz. It shows that 


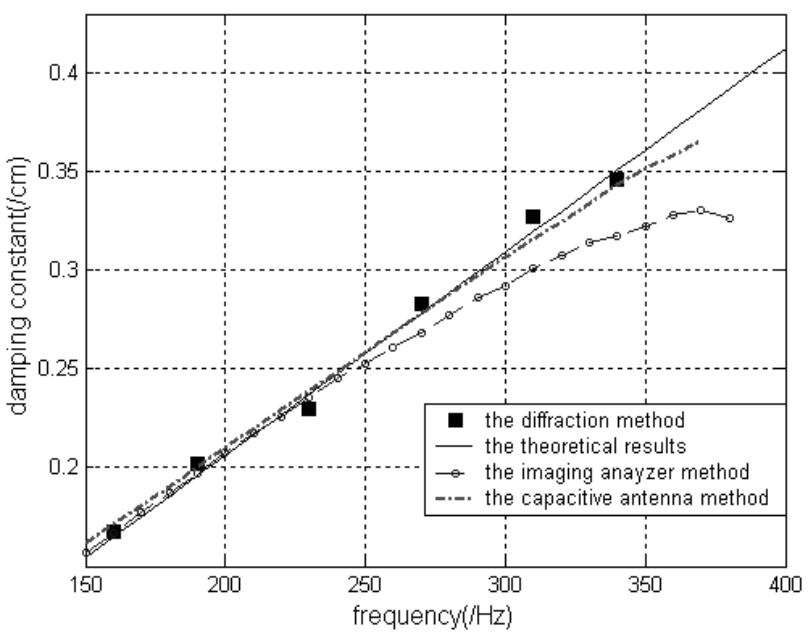

FIG. 6: The discrete dots are experimental data obtained by diffraction method and the solid black line shows the theoretical prediction of Eq. 6.

the damping constant is increasing with the frequency. Then, we give a comparison between the diffraction method and two other methods, i.e., the imaging analyzer method and the capacitive antenna method. In Fig. 6 , it can be seen that at the low frequency, all of them could obtain nearly the same results as the theoretical prediction. However, at the higher frequency, the results of the imaging analyzer method become inaccurate, whereas, the diffraction method and the capacitive antenna method can still get the accurate results. Besides, it should be noticed that the apparatus of the capacitive antenna method is more complicated and expensive.
From the comparison above, we can get the conclusion as follows. The experimental results of the diffraction method are well fitted by the theoretical prediction, and the experiment could be easily performed.

\section{SUMMARY}

The diffraction method has been applied to investigate the proprieties of liquid surface. In our experiment, the stationary diffraction patterns resulting from the LSW (a few hundreds Hertz) were obtained, and the phenomenon was experimentally observed that the intensity distribution of diffraction patterns varies with the position away from the exciter. By analyzing the influence of the amplitude over the intensity distribution, the relationship between the amplitude of LSW and the distance was studied. So a simple method for measuring the damping constant of liquid surface wave is developed, which is based on the analysis of the intensity distribution of diffraction patterns. Besides, according to the Eq. (6), the viscosity of the liquid can also be obtained with this method. It should be noted that the output intensity of the laser beam should be kept steady strictly; otherwise, the inaccurate experimental results may be produced in the experiment. So in our experiment, the laser beam is divided into two sub-beams with a beam splitter. One is used to monitor the laser output stability, and the other is directly incident upon the LSW.

\section{Acknowledgements}

This study was supported by Xi'an Institute of Posts and Telecommunications under Grant No. 101-0424.
[1] B. M. Ocko, X. Z. Wu, E. B. Sirota, S. K. Sinha, and M. Deutsch, Phys. Rev. Lett. 72, 242 (1994).

[2] D. M. Mitrinovic, Z. Zhang, S. M. Williams, Z. Huang, and M. Schlossman, J. Phys. Chem. B 103 (11), 1779 (1999).

[3] K. Sakai, H. Kikuchi, and K. Takagi, Rev. Sci. Instrum. 63(11), 1192 (1991).

[4] J. Penfold and R. K. Thomas, J. Phys.: Condens. Matter 2, 1369 (1990).

[5] L. T. Lee, D. Langevin, and B. Farnoux, Phys. Rev. Lett. 67 , 2678 (1991).

[6] G. Weisbuch, F. Garbay, Am. J. Phys. 47, 355 (1979).

[7] S. Hard, Y. Hamnerius, and O. Nilsson, J. Appl. Phys. 47(6), 2433 (1976).

[8] J. C. Earnshaw, R. C. McGivern, J. Phys. D: Appl. Phys. 20, 82 (1987).

[9] Ka Yee Lee, Tom Chou, Doo Soo Chung et al., J. Phys. Chem. 97(49), 12876 (1993).

[10] M. van den Tempel, R. P. van de Riet, J. Chem. Phys. 42(8), 2796 (1965).

[11] J. D. Barter, K. L. Beach, and P. H. Y. Lee, Rev. Sci. Instrum. 64(9), 2661 (1993).
[12] Q. X. Li, M. Zhao, S. Tang, S. C. Sun, and J. Wu, Appl. Opt. 32(24), 4590 (1993)

[13] J. Chang, R. Wagner, and H. Yuen, J. Fluid Mech. 86, 401 (1978).

[14] P. H. Y. Lee, J. D. Barter, K. L. Beach, et al., SPIE Proc. 1749, 234 (1992).

[15] J. D. Barter, P. H. Y. Lee, Appl. Phys. Lett. 64(15), 1896 (1994).

[16] J. D. Barter, P. H. Y. Lee. Appl. Opt. 36(12), 2630 (1997).

[17] J. D. Barter, Rev. Sci. Instrum. 64, 556 (1993).

[18] J. D. Barter, Phys. Fluids 6(8), 2606 (1994).

[19] R. H. Katyl and U. Ingard, Phys. Rev. Lett. 19, 64 (1967).

[20] J. S. Huang and W. W. Webb, Phys. Rev. Lett. 23, 160 (1969).

[21] K. Y. Lee, T. Chou, D. S. Chung, and E. Mazur, J. Phys. Chem. 97, 12876 (1993).

[22] C. Martel and E. Knobloch, Phys. Rev. E 56, 5544 (1997).

[23] P. Cicuta and I. Hopkinson, Phys. Rev. E 65, 041404 (2002).

[24] P. Cicuta and I. Hopkinson, Colloids Surf. A 233, 97 (2004).

[25] S. Hard, Y. Hamnerius, and O. Nilsson, J. Appl. Phys. 47, 2433 (1976).

[26] K. J. Maloy, J. Feder, and T. Jossang, Rev. Sci. Instrum. 60, 481 (1989). 
[27] B. J. A. Bjorkvik, D. Waaler, and T. Sikkeland, Meas. Sci. Technol. 6, 1572 (1995).

[28] P. Tin, J. A. Mann, W. V. Meyer, and T. W. Taylor, Appl. Opt. 36, 7601 (1997).

[29] J. Dong, R. C. Miao, and J. X. Qi, J. Appl. Phys. 100, 033108 (2006).

[30] R. C. Miao, X. F. Zhao, and J. Shi, Opt. Comm. 259, 592 (2006).

[31] R. C. Miao, Zongli Yang, and Jingtao Zhu, Appl. Phys. Lett. 80(17), 3033 (2002).
[32] J. Lighthill, Waves in fluids, Cambridge University Press, Cambridge, 1978, p.228

[33] D. A. Larson, R. D. Black, M. Green, and R. G. Torti, J. Opt. Soc. Am. A 7, 1745 (1990).

[34] B. D. Duncan, Appl. Opt. 39(17), 2888 (2000).

[35] J. W. Goodman, Introduction to Fourier Optics (McGraw-Hill, San Francisco,1968), p.62

[36] H. Lamb, Hydrodynamics, 6th ed. (Dover, New York, 1932), p.627. 